\title{
Reproducing kernel functions for linear tenth-order bound- ary value problems
}

\author{
Ali Akgül ${ }^{1, *}$, Esra Karatas Akgül ${ }^{2, * *}$, Baris $\operatorname{Orcan}^{3, * * *}$, and Mustafa Inc $^{4, * * * *}$ \\ ${ }^{1}$ Siirt University, Art and Science Faculty, Department of Mathematics, 56100, Siirt/TURKEY \\ ${ }^{2}$ Siirt University, Faculty of Education, Department of Mathematics, 56100, Siirt/TURKEY \\ ${ }^{3}$ Siirt University, Art and Science Faculty, Department of Mathematics, 56100, Siirt/TURKEY \\ ${ }^{4}$ Firat University, Faculty of Science, Department of Mathematics, 23119, Elazig/TURKEY
}

\begin{abstract}
Higher order differential equations have always been an onerous problem to investigate for the mathematicians and engineers. Different numerical methods were applied to get numerical approximations of such problems. This paper gives some reproducing kernel functions to find approximate solutions of the tenth-order boundary value problems (BVPs). These reproducing kernel functions are very important in the reproducing kernel Hilbert space method.
\end{abstract}

\section{Introduction}

A numerical approximation of tenth-order BVPs is rarely presented in the literature [1]. When heating an endless flat film of fluid from below, under the supposition that fluid is subject to the action of rotation and uniform magnetic field across the fluid is applied in the same direction as gravity, unsteadiness starts. When unsteadiness begins in as usual convection, then it can be modeled by tenth-order BVP [2]. Pervaiz et al. [3] introduced the numerical approximations of twelfth-order BVPs by applying non-polynomial cubic spline method. Omotayo et al. [4] presented non-polynomial spline technique for the fourth order BVPs. Usmani [5] introduced his work to approximate the fourth order BVPs by using the quartic spline method. Twizell and Boutayeb [6] enhanced and showed the numerical approximations for higher order value problems. The approximation of second order BVPs was introduced by Alberg and Ito [7]. Siraj-ul-Islam et al [8] introduced a non-polynomial spline method to approximate the sixth-order BVPs. Papamichael and Worsey [9] studied the cubic spline algorithm for solving linear fourth-order BVPs. Siddiqi and Twizell $[10,11]$ enhanced numerical approximations of tenth and twelfth-order BVPs.

\footnotetext{
*e-mail: aliakgu100727@gmail.com

**e-mail: esrakaratas@siirt.edu.tr

***e-mail: barisorcan@gmail.com

****e-mail: minc@firat.edu.tr
} 


\section{MATERIALS AND METHODS}

The main aim of this study is to find some new functions for numerical approximations of linear tenth-order BVPs. We consider

$$
\begin{array}{r}
h^{(x)}(t)+a_{1}(t) h^{(i x)}(t)+a_{2}(t) h^{(v i i i)}(t)+a_{3}(t) h^{(v i i)}(t)+a_{4}(t) h^{(v i)}(t) \\
+a_{5}(t) h^{(v)}(t)+a_{6}(t) h^{(i v)}(t)+a_{7}(t) h^{\prime \prime \prime}(t)+a_{8}(t) h^{\prime \prime}(t) \\
+a_{9}(t) h^{\prime}(t)+a_{10}(t) h(t)=M(t), \quad t \in[a, b],
\end{array}
$$

with boundary conditions:

$$
\begin{aligned}
& h(a)=\alpha_{0}, \quad h(b)=\beta_{0}, \quad h^{\prime \prime}(a)=\alpha_{1}, \quad h^{\prime \prime}(b)=\beta_{1}, \\
& h^{(i v)}(a)=\alpha_{2}, \quad h^{(i v)}(b)=\beta_{2}, \quad h^{(v i)}(a)=\alpha_{3}, \quad h^{(v i)}(b)=\beta_{3}, \\
& h^{(\text {viii })}(a)=\alpha_{4}, \quad h^{(\text {viii })}(b)=\beta_{4},
\end{aligned}
$$

where, $\alpha_{j}, \beta_{j}, j=0,1,2,3,4$ are arbitrary fixed real constants, $a_{j}(t), j=1,2, \ldots, 10$ and $M(t)$ are continuous functions described on $[a, b]$.

We obtain our reproducing kernel functions for following test problem.

\section{Test Problem}

We consider the following tenth-order equation as a second experiment:

$$
h^{(10)}(t)=-\left(80+19 t+t^{2}\right) \exp (t), \quad 0 \leq t \leq 1,
$$

with boundary conditions:

$$
\begin{array}{cl}
h(0)=0, & h(1)=0, \\
h^{2}(0)=0, & h^{2}(1)=-4 \exp (1), \\
h^{4}(0)=-8, & h^{4}(1)=-16 \exp (1), \\
h^{6}(0)=-24, & h^{6}(1)=-36 \exp (1), \\
h^{8}(0)=-48, & h^{8}(1)=-64 \exp (1) .
\end{array}
$$

The exact solution to the above BVP is given by [1]:

$$
h(t)=t(1-t) \exp (t)
$$

\section{Reproducing kernel functions}

We define $V_{2}^{11}[0,1]$ as

$$
V_{2}^{11}[0,1]=\left\{\begin{array}{c}
u, u^{\prime}, u^{\prime \prime}, u^{\prime \prime \prime}, u^{(4)}, u^{(5)}, u^{(6)}, u^{(7)}, u^{(8)}, u^{(9)}, u^{(10)} \quad \text { are absolutely continuous functions, } \\
u^{(11)} \in L^{2}[0,1], \quad u(0)=u^{\prime \prime}(0)=u^{(4)}(0)=u^{(6)}(0)=u^{(8)}(0)=0 \\
u(1)=u^{\prime \prime}(1)=u^{(4)}(1)=u^{(6)}(1)=u^{(8)}(1)=0 .
\end{array}\right\}
$$

We give

$$
\begin{aligned}
\left\langle u, G_{z}\right\rangle_{V_{2}^{11}[0,1]} & =u(0) G_{z}(0)+u^{\prime}(0) G_{z}^{\prime}(0)+u^{\prime \prime}(0) G_{z}^{\prime \prime}(0)+u^{\prime \prime \prime}(0) G_{z}^{\prime \prime \prime}(0)+u^{(4)}(0) G_{z}^{(4)}(0) \\
& +u^{(5)}(0) G_{z}^{(5)}(0)+u^{(6)}(0) G_{z}^{(6)}(0)+u^{(7)}(0) G_{z}^{(7)}(0)+u^{(8)}(0) G_{z}^{(8)}(0) \\
& +u^{(9)}(0) G_{z}^{(9)}(0)+u^{(10)}(0) G_{z}^{(10)}(0)+\int_{0}^{1} u^{(11)}(x) G_{z}^{(11)}(x) d(x) .
\end{aligned}
$$


We have

$$
\begin{gathered}
G_{z}(0)=G_{z}^{\prime \prime}(0)=G_{z}^{(4)}(0)=G_{z}^{(6)}(0)=G_{z}^{(8)}(0)=0 \\
G_{z}(1)=G_{z}^{\prime \prime}(1)=G_{z}^{(4)}(1)=G_{z}^{(6)}(1)=G_{z}^{(8)}(1)=0,
\end{gathered}
$$

by boundary conditions. Therefore, we get

$$
\begin{aligned}
\left\langle u, G_{z}\right\rangle_{V_{2}^{11}[0,1]} & =u^{\prime}(0) G_{z}^{\prime}(0)+u^{\prime \prime \prime}(0) G_{z}^{\prime \prime \prime}(0) \\
& +u^{(5)}(0) G_{z}^{(5)}(0)+u^{(7)}(0) G_{z}^{(7)}(0) \\
& +u^{(9)}(0) G_{z}^{(9)}(0)+u^{(10)}(0) G_{z}^{(10)}(0)+\int_{0}^{1} u^{(11)}(x) G_{z}^{(11)}(x) d(x)
\end{aligned}
$$

We obtain

$$
\begin{aligned}
\left\langle u, G_{z}\right\rangle_{V_{2}^{11}[0,1]} & =u^{\prime}(0) G_{z}^{\prime}(0)+u^{\prime \prime \prime}(0) G_{z}^{\prime \prime \prime}(0)+u^{(5)}(0) G_{z}^{(5)}(0)+u^{(7)}(0) G_{z}^{(7)}(0) \\
& +u^{(9)}(0) G_{z}^{(9)}(0)+u^{(10)}(0) G_{z}^{(10)}(0)+u^{(10)}(1) G_{z}^{(11)}(1)-u^{(10)}(0) G_{z}^{(11)}(0) \\
& -u^{(9)}(1) G_{z}^{(12)}(1)+u^{(9)}(0) G_{z}^{(12)}(0)+u^{(8)}(1) G_{z}^{(13)}(1)-u^{(8)}(0) G_{z}^{(13)}(0) \\
& -u^{(7)}(1) G_{z}^{(14)}(1)+u^{(7)}(0) G_{z}^{(14)}(0)+u^{(6)}(1) G_{z}^{(15)}(1)-u^{(6)}(0) G_{z}^{(15)}(0) \\
& -u^{(5)}(1) G_{z}^{(16)}(1)+u^{(5)}(0) G_{z}^{(16)}(0)+u^{(4)}(1) G_{z}^{(17)}(1)-u^{(4)}(0) G_{z}^{(17)}(0) \\
& -u^{\prime \prime \prime}(1) G_{z}^{(18)}(1)+u^{\prime \prime \prime}(0) G_{z}^{(18)}(0)+u^{\prime \prime}(1) G_{z}^{(19)}(1)-u^{\prime \prime}(0) G_{z}^{(19)}(0) \\
& -u^{\prime}(1) G_{z}^{(20)}(1)+u^{\prime}(0) G_{z}^{(20)}(0)+u(1) G_{z}^{(21)}(1)-u(0) G_{z}^{(21)}(0) \\
& -\int_{0}^{1} u(x) G_{z}^{(22)}(x) d(x)
\end{aligned}
$$


by integration by parts. Then, we have

$$
\begin{aligned}
\left\langle u, G_{z}\right\rangle_{V_{2}^{11}[0,1]} & =u^{\prime}(0) G_{z}^{\prime}(0)+u^{\prime \prime \prime}(0) G_{z}^{\prime \prime \prime}(0)+u^{(5)}(0) G_{z}^{(5)}(0)+u^{(7)}(0) G_{z}^{(7)}(0) \\
& +u^{(9)}(0) G_{z}^{(9)}(0)+u^{(10)}(0) G_{z}^{(10)}(0)+u^{(10)}(1) G_{z}^{(11)}(1)-u^{(10)}(0) G_{z}^{(11)}(0) \\
& -u^{(9)}(1) G_{z}^{(12)}(1)+u^{(9)}(0) G_{z}^{(12)}(0)-u^{(7)}(1) G_{z}^{(14)}(1)+u^{(7)}(0) G_{z}^{(14)}(0) \\
& -u^{(5)}(1) G_{z}^{(16)}(1)+u^{(5)}(0) G_{z}^{(16)}(0)-u^{\prime \prime \prime}(1) G_{z}^{(18)}(1)+u^{\prime \prime \prime}(0) G_{z}^{(18)}(0) \\
& -u^{\prime}(1) G_{z}^{(20)}(1)+u^{\prime}(0) G_{z}^{(20)}(0)-\int_{0}^{1} u(x) G_{z}^{(22)}(x) d(x)
\end{aligned}
$$

We have

$$
\begin{aligned}
& G_{z}^{\prime}(0)+G_{z}^{(20)}(0)=0, \quad G_{z}^{\prime \prime \prime}(0)+G_{z}^{18}(0)=0 \\
& G_{z}^{(5)}(0)+G_{z}^{16}(0)=0, \quad G_{z}^{(7)}(0)+G_{z}^{14}(0)=0 \\
& G_{z}^{(9)}(0)+G_{z}^{12}(0)=0, \quad G_{z}^{10}(0)-G_{z}^{11}(0)=0 \\
& G_{z}^{(11)}(1)=0, \quad G_{z}^{(12)}(1)=0, \quad G_{z}^{(14)}(1)=0 \\
& G_{z}^{(16)}(1)=0, \quad G_{z}^{(18)}(1)=0, \quad G_{z}^{(20)}(1)=0
\end{aligned}
$$

Thus, we acquire

$$
\left\langle u, G_{z}\right\rangle_{V_{2}^{11}[0,1]}=-\int_{0}^{1} u(x) G_{z}^{(22)}(x) d(x)=u(z) .
$$

Therefore, we obtain

$$
G_{z}(x)=\left\{\begin{array}{lll}
\sum_{i=1}^{11} c_{i} x^{i-1} & , & x \leq z \\
\sum_{i=1}^{11} d_{i} x^{i-1} & , & x>z
\end{array}\right.
$$

by

$$
G_{z}^{(22)}(x)=-\delta(x-z)
$$




\section{Conclusion}

We obtained some reproducing kernel functions to find approximate solutions of the tenthorder boundary value problems in this paper. We need these reproducing kernel functions for applications of the reproducing kernel Hilbert space method.

\section{References}

[1] M. J. Iqbal , S. Rehman, A. Pervaiz, and A. Hakeem, Approximations for Linear Tenthorder Boundary Value Problems through Polynomial and Non-polynomial Cubic Spline Techniques, Proceedings of the Pakistan Academy of Sciences 52 (4): 389-396 (2015).

[2] Chandrasekhar. Hydrodynamic and Hydromagnetic Stability. Clarendon Press, Oxford, England (1961).

[3] Pervaiz, A., Zafar, Z. \& M.O. Ahmad. A non-polynomial spline method for solving linear twelfth order boundary-value problems. Pakistan Academy of Sciences 51(2): 157-165 (2014).

[4] Omotayo, A.T. \& O.M. Ogunlaran. A non-polynomial spline method for solving linear fourth order boundary-value problems. International Journal of the Physical Sciences 6: 3246-3254 (2011).

[5] Usmani, R.A. The use of quartic splines in the numerical solution of the fourth order boundary value problem. Journal of Computational and Applied Mathematics 44:187-199 (1992).

[6] Twizell, E.H. \& A. Boutayeb. Numerical methods for eight, tenth and twelfth order eigenvalue problems arising in thermal instability. Advances in Computational Mathematics 2:407-436 (1994).

[7] Alberg, J.H. \& T. Ito. A collocation method for two-point boundary value problems. Mathematics of Computation 29: 761-776 (1975).

[8] Siraj-ul-Islam, I.A. Tirmizi, Fazal-i-Haq \& M.A. Khan. A non-polynomial spline approach to the solution of sixthorder BVPs. Applied Mathematics and Computation 195: 277-284 (2008).

[9] Papamichael, N. \& A.J. Worsey. A cubic spline method for the solution of linear fourthorder boundary value problems. Journal of Computational and Applied Mathematics 7: 187-189 (1981).

[10] Siddiqi, S.S. \& E.H. Twizell. Spline solution of linear twelfth-order boundary value problems. Journal of Computational and Applied Mathematics 78: 371-390 (1997).

[11] Siddiqi, S.S. \& E.H. Twizell. Spline solution of linear tenth-order boundary value problems. Journal of Computational and Applied Mathematics 68: 345-362 (1998).

[12] Siddiqi, S.S. \& Ghazala Akram. Spline solutions of tenth-order boundary value problems. Journal of Computational and Applied Mathematics 185: 115-127 (2007). 